\title{
APPROXIMATING RATIONAL SPACES WITH ELLIPTIC COMPLEXES AND A CONJECTURE OF ANICK
}

\author{
Barry Jessup And Aniceto Murillo-Mas ${ }^{1}$
}

\begin{abstract}
An elliptic space is one whose rational homotopy and rational cohomology are both finite dimensional. David Anick conjectured that any simply connected finite CW-complex $S$ can be realized as the $k$-skeleton of some elliptic complex as long as $k>\operatorname{dim} S$. A functorial version of this conjecture due to McGibbon is that for any $n$ there exists an elliptic complex $E_{n}$ and an $n$-equivalence $S \rightarrow E_{n}$. In fact, this is equivalent to its Eckmann-Hilton dual, which we prove in the rational category for a small class of simply connected spaces. Moreover, we construct the $n$-equivalence in such a way that the homotopy fibre is, rationally, a product of a finite number of odd spheres.
\end{abstract}

\section{Introduction.}

A topological space is elliptic if its rational homotopy and rational cohomology are both finite dimensional. This class includes many interesting and well-known spaces which enjoy important structural properties: For example, their Euler characteristic is non negative, their homotopy Euler characteristic is non-positive, and their rational cohomology satisfies Poincaré duality $[\mathbf{H}]$. Indeed, Halperin and Friedlander have shown how to characterize them in terms of the degrees of a basis for their rational homotopy $[\mathbf{F H}]$. Moreover, a result of McGibbon and Wilkerson $[\mathbf{M W}]$ asserts that an elliptic space has an exponent for almost every prime number. The latter is a partial answer to the conjecture of Moore that a simply connected space is elliptic if and only if has an exponent for every prime number [S].

Elliptic spaces seem very special, and rightly so, as the generic space is not elliptic. Thus one might not expect to them to exhibit a sufficient variety of behaviour to be useful in approximating general spaces. However, Anick has conjectured the following:

Conjecture (Anick). Any simply connected finite $C W$-complex $S$ is the $k$-skeleton of an elliptic complex as long as $k \geq \operatorname{dim} S$.

\footnotetext{
${ }^{1}$ Partially supported by a DGICYT grant (PB94-1485)
} 
Of course, skeleta are not homotopy invariants, and McGibbon has suggested that this conjecture can be most profitably viewed in the following terms $[\mathrm{M}]$.

Definition. A space $S$ can be approximated arbitrarily closely on the right (resp. left) by a class of spaces $\mathcal{A}$ if for each natural number $n$ there is a space $A_{n} \in \mathcal{A}$ and an $n$-equivalence $S \rightarrow A_{n}$ (resp. $A_{n} \rightarrow S$ ).

Naturally, the definition depends on the notion of " $n$-equivalence", and we will henceforth always work in a subcategory of the category $\mathrm{CW}_{1}$ of 1-connected spaces with the homotopy type of a CW complex.

Now let $\mathcal{A}$ and $\mathcal{T}$ be any two classes of spaces and consider the following (Eckmann-Hilton dual) conjectures (a "Postnikov piece" is a space with finitely generated homotopy):

Conjecture $R_{\mathcal{A}}(\mathcal{T})$. Any finite complex $S \in \mathcal{T}$ may be approximated arbitrarily closely on the right by the class $\mathcal{A}$.

Conjecture $L_{\mathcal{A}}(\mathcal{T})$. Any Postnikov piece $S \in \mathcal{T}$ may be approximated arbitrarily closely on the left by the class $\mathcal{A}$.

In fact, in $\mathrm{CW}_{1}$ (or in any subcategory where equivalence $=$ homology equivalence $=$ homotopy equivalence), $R_{\mathcal{A}}(\mathcal{T})$ and $L_{\mathcal{A}}(\mathcal{T})$ are equivalent for any choice of $\mathcal{A}$ and for any $\mathcal{T}$ which is closed (up to homotopy) under the operations of taking $k$-skeletons and $k$-Postnikov stages (e.g. $\mathcal{T}=\mathcal{C} \mathcal{W}_{1}$, all 1-connected CW complexes). We present a proof of this in Theorem 2.1. Moreover, an interesting example of these conjectures is presented in Theorem 2.3, where it is shown that in $\mathrm{CW}_{1}$, any finite complex can be approximated arbitrarily closely on the left and hence on the right (see Remark 2.2 below) by the class $\mathcal{V}$ of simply connected, smooth, closed manifolds, so that both $R_{\mathcal{V}}\left(\mathcal{C W}_{1}\right)$ and $L_{\mathcal{V}}\left(\mathcal{C W}_{1}\right)$ hold. These results were communicated to the authors by Charles McGibbon and we thank him for his interest and guidance.

A functorial version of Anick's conjecture is then $R_{\mathcal{E}}\left(\mathcal{C W}_{1}\right)$, where $\mathcal{E}$ denotes the class of simply connected elliptic spaces. In this case, these conjectures are most naturally viewed in the full subcategory $\mathcal{Q}$ of simply connected rational spaces with finite type (rational) homology, and in this setting $L_{\mathcal{E}}$ and $R_{\mathcal{E}}$ are known to be true in many examples. In particular, if

$$
\mathcal{T}=\left\{S \mid \pi_{*}(S)=\pi_{\text {odd }}(S)\right\}
$$

$\operatorname{dim} \pi_{*}(S)<\infty$ guarantees that $S$ is already elliptic and so $L_{\mathcal{E}}(\mathcal{T})$ is trivially true. Dually, if

$$
\mathcal{T}^{\prime}=\left\{S \mid H_{*}(S)=H_{\text {odd }}(S)\right\}
$$


and $S$ is a finite complex then $S$ is a finite wedge of odd spheres and so any (rational) $n$-Postnikov stage is elliptic, i.e., $R_{\mathcal{E}}\left(\mathcal{T}^{\prime}\right)$ holds. It is also implicit in the proof of Proposition 2 in [H2] that if we restrict ourselves to those $S$ where $H^{*}(S)$ is Noetherian, $L_{\mathcal{E}}$ is true, and moreover, the approximation $E_{n} \rightarrow S$ can be chosen so its homotopy fibre is, rationally, a product of Krull-dim $H^{*}(S)$ odd spheres.

The main result of this paper is that we prove conjecture $L_{\mathcal{E}}(\mathcal{M})$ in the category $\mathcal{Q}$ for the following class $\mathcal{M}$ of spaces:

Definition. A space $S$ belongs to $\mathcal{M}$ if there is a fibration $G \rightarrow S \stackrel{p}{\rightarrow} B$ in which

(i) $\pi_{*}(p)$ is onto,

(ii) $\pi_{\text {even }}(B)=0$, and

(iii) the Hurewicz map $h \pi_{*}(G) \rightarrow H_{*}(G)$ is injective in even degrees.

Observe that a space $S$ for which $\operatorname{dim} \sum_{k} \pi_{2 k}(S) \leq 1$ is in $\mathcal{M}$. Moreover, if $S \in \mathcal{M}$ and $S \stackrel{f}{\rightarrow} T$ is a fibration in which $\pi_{*}(f)$ is surjective, then $T$ is in $\mathcal{M}$ as well. In the next section, we'll see that one can describe $\mathcal{M}$ quite simply in terms of Sullivan minimal models.

We make no assumptions on $H^{*}(S)$ when we prove:

Theorem 1. $L_{\mathcal{E}}(\mathcal{M})$ holds in $\mathcal{Q}$. Moreover, $E_{n} \rightarrow S$ can be chosen so that its homotopy fibre is a product of a finite number of odd (rational) spheres.

As we shall see, a consequence of the proof of Theorem 1 is:

Corollary. If $G \rightarrow S \stackrel{p}{\rightarrow} B$ is a fibration that guarantees $S \in \mathcal{M}$, and $\pi_{>N}(S)=0$, then the number of odd spheres in the fibre of $E_{n} \rightarrow S$ is bounded above by

$$
\operatorname{dim} \pi_{\text {even }}(G) \cdot[n / 2(N / 2-1)]^{\operatorname{dim} \pi(B)} .
$$

Our result is reminiscent of the theorem of Halperin and Levin $[\mathbf{H L}]$, Thm. C, by which a simply connected space $B$ with finite dimensional rational homotopy is the base of a fibration $F \rightarrow E \stackrel{p}{\rightarrow} B$ where both $F$ and $E$ have finite dimensional rational cohomology and $\pi_{\text {even }}(E) \otimes \mathbb{Q}=0$. This last property of $E$ shows that $p$ cannot, in general, induce an isomorphism in rational homotopy groups up to any degree fixed in advance.

This paper is organized as follows. In the next section we shall first present a proof of the equivalence $R_{\mathcal{A}}(\mathcal{T}) \Longleftrightarrow L_{\mathcal{A}}(\mathcal{T})$ mentioned above and establish these conjectures in $\mathrm{CW}_{1}$ when $\mathcal{A}$ is the class of smooth, closed manifolds. 
In Section 3, we move into the rational category, recalling some basic facts in rational homotopy, and prove Theorem 1 in the algebraic context of Sullivan's minimal models in Proposition 3.1. Some examples and open questions are given in the final section.

\section{Duality and approximation by manifolds.}

We remind the reader that in this section, we are working in the category of spaces with the homotopy types of 1-connected CW complexes.

In what follows, we denote the inclusion of the $k$-skeleton of a CW complex $Y$ by $Y_{k} \stackrel{i_{k}}{\longrightarrow} Y$. The $k$-th stage of the (CW-) Postnikov decomposition of $Y$ will be written $Y \stackrel{f^{k}}{\longrightarrow} Y^{k}$, so that $f^{k}$ is a $k$-equivalence and $\pi_{>k}\left(Y^{k}\right)=0$. Note that $i_{k+1}$ is a $k$-equivalence which induces a homotopy equivalence $\left(Y_{k+1}\right)^{k} \simeq Y^{k}$ and that we may choose $\left(Y^{k}\right)_{j}=Y_{j}$ for $0 \leq j \leq k+1$.

Theorem $2.1[\mathrm{M}]$. If $\mathcal{A}$ and $\mathcal{T}$ are any classes of spaces in $C W_{1}$ such that, up to homotopy, $\mathcal{T}$ contains its $k$-skeletons and $k$-Postnikov stages (for $k$ sufficiently large), then $R_{\mathcal{A}}(\mathcal{T}) \Longleftrightarrow L_{\mathcal{A}}(\mathcal{T})$.

Proof. We first show that $R_{\mathcal{A}}(\mathcal{T}) \Rightarrow L_{\mathcal{A}}(\mathcal{T})$. Suppose $S \in \mathcal{T}$ satisfies $\pi_{\geq N} S=$ 0 , and let $n \in \mathbb{N}$ be given. If $m=\max \{n, N\}, S \simeq S^{m}$, so that remarks just above show that there exists a homotopy equivalence $\left(S_{m+1}\right)^{m} \simeq S$. Since we may suppose that $S_{m+1} \in \mathcal{T}, R_{\mathcal{A}}(\mathcal{T})$ implies that there is $A \in \mathcal{A}$ and an $m$-equivalence $S_{m+1} \rightarrow A$. The latter induces a homotopy equivalence $\left(S_{m+1}\right)^{m} \simeq A^{m}$, so that the composition $A \stackrel{f^{m}}{\longrightarrow} A^{m} \simeq\left(S_{m+1}\right)^{m} \simeq S$ is an $m$-equivalence establishing $L_{\mathcal{A}}(\mathcal{T})$.

To see that $L_{\mathcal{A}}(\mathcal{T}) \Rightarrow R_{\mathcal{A}}(\mathcal{T})$, assume $S \in \mathcal{T}$ satisfies $H^{\geq N} S=0$, let $n \in \mathbb{N}$ be given and set $m=\max \{n, N\}$. In this case, we may suppose that $S=S_{m}$. Using $L_{\mathcal{A}}(\mathcal{T})$ and the fact that $\mathcal{T}$ contains its $m$-Postnikov stages, choose an $m$-equivalence $A \stackrel{h}{\rightarrow} S^{m}$ where $A \in \mathcal{A}$, so that $A^{m} \stackrel{h^{m}}{\rightarrow}\left(S^{m}\right)^{m}=$ $S^{m}$ is a homotopy equivalence. This means that we may choose cellular decompositions of $A$ and $S$ in which $A_{m+1}=\left(A^{m}\right)_{m+1}=\left(S^{m}\right)_{m+1}=S_{m+1}$. The composition $S=S_{m+1}=A_{m+1} \rightarrow A$ is then an $m$-equivalence which proves $R_{\mathcal{A}}(\mathcal{T})$.

Remark 2.2. Let $A$ and $S$ be simply-connected CW complexes and let $R(S)$ (resp. $L(S)$ ) be the statement "there exists an $m$-equivalence $S \rightarrow A$ " (resp. "there exists an $m$-equivalence $A \rightarrow S$ "). Observe that the proof of theorem above lies in the equivalence $L\left(S^{m}\right) \Longleftrightarrow R\left(S_{m+1}\right)$. On the other hand if $X \rightarrow S_{m+1}$ (resp. $S^{m} \rightarrow X$ ) is an $m$-equivalence so is $X \rightarrow$ $S_{m+1} \stackrel{i_{m+1}}{\longrightarrow} S \stackrel{f_{m}}{\longrightarrow} S^{m}$ (resp. $S_{m+1} \stackrel{i_{m+1}}{\longrightarrow} S \stackrel{f_{m}}{\longrightarrow} S^{m} \rightarrow X$ ). Hence we have 
the following chain of implications

$$
L\left(S_{m+1}\right) \Rightarrow L\left(S^{m}\right) \Longleftrightarrow R\left(S_{m+1}\right) \Leftarrow R\left(S^{m}\right) .
$$

In particular, if $X$ is a finite, simply-connected CW complex and $m>\operatorname{dim} X$, then $L(X) \Rightarrow R(X)$. Dually, if $X$ is an m-Postnikov piece, $R(X) \Rightarrow L(X)$.

As before, let $\mathcal{V}$ denote the class of closed, compact, simply-connected manifolds.

Theorem $2.3[\mathrm{Br}]$, [Sp]. In $C W_{1}, R_{\mathcal{V}}\left(\mathcal{C W}_{1}\right)$ (and hence $\left.L_{\mathcal{V}}\left(\mathcal{C} \mathcal{W}_{1}\right)\right)$ holds.

Proof. We prove that every finite complex X can be approximated arbitrarily closely on the left by $\mathcal{V}$. Remark 2.2 will then imply that $R_{\mathcal{V}}\left(\mathcal{C W}_{1}\right)$ holds. Let $n=\operatorname{dim} X$ and note that $X$ has the homotopy type of a simplicial complex and can therefore be embedded in $\mathbb{R}^{n+k}$, for $k \geq n+1$. If $m$ is a given integer, choose $k>m+2$. Let $N$ be a regular neighborhood of $K$ and $\partial N \hookrightarrow N$ the inclusion of its boundary. From a general position argument it follows that the morphism induced by the inclusion $\pi_{i}(N-X) \rightarrow \pi_{i}(N)$ is an isomorphism for $i<k-1$ and it is onto for $i=k-1$. Now, since $N$ is a regular neighborhood, $\partial N \hookrightarrow N-X$ is a homotopy equivalence and therefore $\pi_{i}(\partial N) \rightarrow \pi_{i}(N)$ is an isomorphism for $i<k-1$. Finally, choose an equivalence $N \stackrel{\simeq}{\rightarrow} X$ and observe that $\partial N \hookrightarrow N \stackrel{\simeq}{\longrightarrow} X$ is an $m$-equivalence.

The above proof appears in [Br, Thm. I.4.4], although W. Browder refers to $[\mathbf{S p}]$ where this is done in a slightly different way. Spivak shows that the homotopy fibre of the inclusion $\partial N \hookrightarrow N$ is $k-1$-connected. Note that this shows that the approximation can be done on the left even when the complex is not simply-connected.

\section{Towards Anick's conjecture in the Rational category.}

We work with $\mathbb{Q}$ as ground field and we alert the reader that henceforth, all our spaces are simply connected rational spaces with the homotopy type of CW complexes with rational cohomology of finite type. We refer the reader to $[\mathrm{BG}],[\mathbf{H 1}]$ and $[\mathrm{Su}]$ for the basic material on Sullivan models.

A commutative graded differential algebra (hereafter cgda) $\left(A, d_{A}\right)$ is $c$ connected if $H^{0}\left(A, d_{A}\right) \cong \mathbb{Q}$. A morphism $\phi:\left(A, d_{A}\right) \stackrel{\simeq}{\rightarrow}\left(B, d_{B}\right)$ is a quasiisomorphism if $H^{*} \phi$ is an isomorphism. If $\left(A, d_{A}\right) \stackrel{\phi}{\rightarrow}\left(B, d_{B}\right)$ is a morphism of c-connected cgdas, a Sullivan Model of $\phi$ is a factoring of $\phi=\psi i$ in $\left(A, d_{A}\right) \stackrel{i}{\rightarrow}(A \otimes \Lambda X, d) \stackrel{\psi}{\rightarrow}\left(B, d_{B}\right)$ where $\psi$ is a quasi-isomorphism, $i(a)=$ $a \otimes 1$ for $a \in A$ and $\Lambda X$ denotes the free commutative graded algebra on the 
graded vector space $X=\sum_{n \geq 0} X^{n}$ which has a well ordered, homogeneous basis $\left\{x_{\alpha} \mid \alpha \in I\right\}$ such that

(1) $d x_{\alpha} \in A \otimes \Lambda X_{<\alpha}$ and

(2) $\alpha<\beta \Longrightarrow \operatorname{deg} \alpha \leq \operatorname{deg} \beta$.

Here, $X_{<\alpha}$ denotes $\operatorname{span}\left\{x_{\beta} \mid \beta<\alpha\right\}$. If $X=X^{\geq 2}$, (1) and (2) are equivalent to $d x_{\alpha} \in \Lambda^{\geq 2}\left(X_{<\alpha}\right)$. Such a basis is called a KS-basis. The factoring is determined up to isomorphism by $\phi$ and we say that $i$ represents $\phi$.

The projection $(A \otimes \Lambda X, d) \stackrel{\rho}{\rightarrow} A \otimes \Lambda X / I \cong \Lambda X$ (in which $I$ denotes the differential ideal generated by the augmentation ideal of $A$ ) induces a differential $\tilde{d}$ in $\Lambda X$ and the sequence $\left(A, d_{A}\right) \stackrel{i}{\rightarrow}(A \otimes \Lambda X, d) \stackrel{\rho}{\rightarrow}(\Lambda X, \tilde{d})$ or sometimes just $\left(A, d_{A}\right) \stackrel{i}{\rightarrow}(A \otimes \Lambda X, d)$ is called a minimal KS-extension.

Sullivan defined a contravariant functor $\mathcal{A}$ which associates to each space $S$ a cgda $\mathcal{A}(S)$ over $\mathbb{Q}$ which computes the rational homotopy of $S$. A Sullivan minimal model of $(\mathbb{Q}, 0) \rightarrow \mathcal{A}(S)$ is of the form $(\mathbb{Q}, 0) \rightarrow(\Lambda X, d) \rightarrow \mathcal{A}(S)$ and $(\Lambda X, d)$ is the Sullivan minimal model of $\mathrm{S}$. If $S \rightarrow T$ is a continuous map then a standard lifting lemma applied to $\mathcal{A}(f)$ gives a unique homotopy class of morphisms between their minimal models, any of which is called a Sullivan representative of $f$.

The minimal model of $S$ carries the rational homotopy type of $S$, that is, the homotopy type of its localization at the rationals $S_{\mathbb{Q}}$ : In particular, as graded vector spaces, $X^{n}$ is the dual of $\pi_{n}(S) \otimes \mathbb{Q}$ and this isomorphism can be described as follows. Let $A_{n}=\left(\Lambda u / u^{2}, 0\right)$ be generated in degree $n$. Since there is a quasi-isomorphism $\mathcal{A}\left(S^{n}\right) \rightarrow A_{n}$, this is a cgda with the homotopy type of an $n$-sphere. Given $f: S^{n} \rightarrow S$, we have $\mathcal{A}(f):(\Lambda X, d) \rightarrow A_{n}$ (by composition on the right and the left). Thus to any $x \in X$ one associates the homomorphism $\phi_{x}: \pi_{n}(S) \rightarrow \mathbb{Q}$ defined by $\phi_{x}([f])=$ the coefficient of $u$ in $\mathcal{A}(f)(x)$.

The rational dual of the Hurewicz map $h: \pi_{*}(S) \rightarrow H_{*}(S)$ is induced by $\Lambda X \rightarrow \Lambda X / \Lambda^{\geq 2} X \cong X$. Hence, whenever $h: \pi_{*}(S) \rightarrow H_{*}(S)$ is injective, $h^{*}$ is surjective. In particular, any minimal model $(\Lambda X, d)$ of the fibre $F$ in the assumptions of Theorem 1 will satisfy $d X^{\text {even }}=0$.

Given a Serre fibration : $F \stackrel{i}{\rightarrow} E \stackrel{p}{\rightarrow} B$, there is a commutative diagram of augmented cgda's

$$
\begin{array}{llll} 
& \mathcal{A}(B) \stackrel{\mathcal{A}(p)}{\longrightarrow} & \mathcal{A}(E) \quad \stackrel{\mathcal{A}(i)}{\longrightarrow} \mathcal{A}(F) \\
\simeq \uparrow \phi_{B} & \simeq \uparrow & \uparrow \alpha \\
(\Lambda X, d) \longrightarrow & (\Lambda X \otimes \Lambda Y, d) \longrightarrow(\Lambda Y, \tilde{d})
\end{array}
$$


in which $(\Lambda X, d)$ is a Sullivan model for $B$ and the bottom row is the minimal model of $\mathcal{A}(p) \circ \phi_{B}$. If $\alpha:(\Lambda Y, \tilde{d}) \rightarrow \mathcal{A}(F)$ is a quasi-isomorphism, $\xi$ is called a rational fibration. In particular, if $B$ is simply connected, $\xi$ is a rational fibration $[\mathbf{G}]$. We call the bottom row of this diagram a minimal KS-extension associated to the fibration. Note that in general, the middle CGDA need not be a minimal model of $E$. It will be, precisely when the image of the connecting homomorphism $\partial: \pi_{k}(B) \rightarrow \pi_{k-1}(F)$ is strictly torsion. In this case one can choose the linear part of $d y$ to be zero for all $y \in Y($ see $[\mathbf{H} \mathbf{1}])$.

Observe that a space $S \in \mathcal{M}$ if and only if has a minimal model of the form $(\Lambda Y \otimes \Lambda X, d)$ in which $\operatorname{dim}(Y \oplus X)<\infty, Y=Y^{\text {odd }},(\Lambda Y, d)$ is a subcomplex and $d X^{\text {even }} \subset \Lambda^{+} Y \otimes \Lambda X$.

Thus $L_{\mathcal{E}}(\operatorname{obj} \mathcal{Q})$ has the following equivalent formulation in terms of Sullivan models (we include the non-simply connected case here):

Conjecture $L_{\mathcal{E}}(\operatorname{obj} \mathcal{Q})$. Let $(\Lambda V, d)$ be a connected $\left(V^{0}=0\right)$ minimal model in which $V$ is finite dimensional. Then, for any $n \in \mathbb{N}$ there exists an elliptic minimal model $(\Lambda(V \oplus W), d) \cong(\Lambda V \otimes \Lambda W, d)$ which is a KSextension of $(\Lambda V, d)$ with $W=W^{>n}$.

We require $(\Lambda(V \oplus W), d)$ to be a minimal model, not just a minimal KSextension, so that none of the rational homotopy $(V)$ of $\Lambda V$ is lost. Otherwise one can proceed as in [HL, Thm. 3.1] to get a minimal KS-extension (but not a minimal model) $\Lambda V \rightarrow \Lambda V \otimes \Lambda Y$ in which the minimal model of this complex is generated by an oddly graded vector space. However, one 'loses' all of $V^{\text {even }}$ in this way. Also note that in this method, the degrees of the new generators cannot be chosen to be bounded below by some integer fixed in advance.

Theorem 1 is now clearly a consequence of the following:

Proposition 3.1. Let $(\Lambda Y, d) \rightarrow(\Lambda Y \otimes \Lambda X, d)$ be a minimal $K S$ extension of minimal models such that $\operatorname{dim}(Y \oplus X)<\infty, Y=Y^{\text {odd }}$ and $d X^{\text {even }} \subset$ $\Lambda^{+} Y \otimes \Lambda X$. Then, given any integer $N$, there exists a minimal $K S$ extension

$$
(\Lambda Y \otimes \Lambda X, d) \rightarrow(\Lambda Y \otimes \Lambda X \otimes \Lambda Z, d) \rightarrow(\Lambda Z, 0)
$$

in which $(\Lambda Y \otimes \Lambda X \otimes \Lambda Z, d)$ is an elliptic minimal model and $Z=Z^{\text {odd }}=$ $Z^{\geq N}$.

Recall [B-L], [T] that a Quillen model of a space $S$ is a differential graded Lie algebra $(\mathbb{L}(V), \partial)$, which is free as graded Lie algebra over the graded vector space $V$ and is such that the cdga of cochains on $(\mathbb{L}(V), \partial)$ and $\mathcal{A}(S)$ 
have the same homotopy type. The homology $H_{*}(\mathbb{L}(V), \partial)$ of such a model is then isomorphic to the rational homotopy Lie algebra $\pi_{*}(\Omega S) \otimes \mathbb{Q}$ of $S$. Moreover, any simply connected complex $S$ has a Quillen model that reflects its cellular decomposition, i.e., a model $(\mathbb{L}(V), \partial)$ in which $V$ is the graded vector space with the cells basis and where $\partial$ describes the attaching maps. Thus the conjecture $R_{\mathcal{E}}$ may restated as: For any minimal Quillen model $(\mathbb{L}(V), \partial)$ in which $\operatorname{dim} V<\infty$, there is an embedding into a minimal Quillen model $(\mathbb{L}(V), \partial) \hookrightarrow(\mathbb{L}(V \oplus W), \partial)$ in which both $W$ and $H_{*}(\mathbb{L}(V \oplus W), \partial)$ are finite dimensional.

Before beginning the proof of Proposition 3.1, we give a brief explanation of our strategy. First recall that a minimal KS-extension of the form

$$
(\Lambda u, 0) \rightarrow(\Lambda u \otimes \Lambda V, d) \rightarrow(\Lambda V, \bar{d}),
$$

with $u$ of odd degree, induces a derivation $\theta$ on $(\Lambda V, \bar{d})$, of degree $1-|u|$, defined by:

$$
d \Phi=\bar{d} \Phi+u \theta(\Phi), \quad \Phi \in \Lambda V .
$$

This is the holonomy derivation. Observe that, for reasons of degree, $\theta$ is locally nilpotent, i.e., given $\Phi \in \Lambda V$ there exists an integer $n$ (depending on $\Phi)$ such that $\theta^{n}(\Phi)=0$.

Let $(\Lambda Y, d) \rightarrow(\Lambda Y \otimes \Lambda X, d)$ be the extension in the statement of Proposition 3.1. Our proof will proceed by induction on $\operatorname{dim} Y$ as follows: Let $Y=\langle y\rangle \oplus U$, and consider the K-S extension $(\Lambda y, 0) \rightarrow(\Lambda Y \otimes \Lambda X, d) \rightarrow$ $(\Lambda U \otimes \Lambda X, d)$, with the associated holonomy derivation $\theta$ defined on the fibre $(\Lambda U \otimes \Lambda X, \bar{d})$. The inductive hypothesis will yield an elliptic extension $\left(\Lambda U \otimes \Lambda X \otimes \Lambda Z^{\prime}, \bar{d}\right)$. We then need to extend $\theta$ to $Z^{\prime}$, but to ensure that it commutes with the differential, it will be necessary to add (possibly) more odd generators. The derivation $\theta$ is always locally nilpotent, so that we will only need a finite number of new generators at this point, and, with some care, we will always be able to bound their degrees away from $N$. We then use the extended $\theta$ to define, via $(*)$, the differential for $\Lambda Y \otimes \Lambda X \otimes \Lambda Z$, which will also be elliptic.

It will become evident later in the proof that the lower bound for the degrees of the new generators is essentially due to the following:

Remark 3.2. Let $\theta$ be a derivation of the model $\Lambda V$. Observe that $\theta\left(\Lambda^{\geq n} V\right) \subset \Lambda^{\geq n} V$. Hence, if $V$ is $p$-connected $\left(V=V^{\geq p}\right)$ and $\Phi \in \Lambda^{\geq n} V$ is such that $\theta(\Phi) \neq 0$ then $|\theta(\Phi)| \geq n p$. This is also true if we replace $\theta$ by $\theta^{k}$ for any $k \geq 1$.

Proof of Proposition 3.1. Let $n=\operatorname{dim} Y$ and $p=\operatorname{dim} X^{\text {even }}$. It will be convenient (although not too standard) to choose a homogeneous ba- 
sis $\left\{y_{n}, \ldots, y_{1}\right\}$ of $Y$ so that $d y_{m} \in \Lambda\left(y_{n}, \ldots, y_{m+1}\right)$. For each $m$ between 1 and $n$, consider the KS-extensions

$$
\begin{gathered}
\left(\Lambda\left(y_{n}, \ldots, y_{m+1}\right) \otimes \Lambda X, d\right) \rightarrow(\Lambda Y \otimes \Lambda X, d) \rightarrow\left(\Lambda\left(y_{m}, \ldots, y_{1}\right) \otimes \Lambda X, d_{m}\right) \\
\left(\Lambda y_{m}, 0\right) \rightarrow\left(\Lambda\left(y_{m}, \ldots, y_{1}\right) \otimes \Lambda X, d_{m}\right) \rightarrow\left(\Lambda\left(y_{m-1}, \ldots, y_{1}\right) \otimes \Lambda X, d_{m-1}\right)
\end{gathered}
$$

and write $d_{m}=d_{m-1}+y_{m} \otimes \theta_{m}$, as in $(*)$.

The inductive assumption is the following: For each $m(\leq \operatorname{dim} Y)$ there exists an elliptic KS extension

$$
\left(\Lambda\left(y_{m}, \ldots, y_{1}\right) \otimes \Lambda X, d_{m}\right) \hookrightarrow\left(\Lambda\left(y_{m}, \ldots, y_{1}\right) \otimes \Lambda X \otimes \Lambda Z_{m}, d_{m}\right)
$$

which is minimal as a model, together with a well-ordered homogeneous basis $\mathcal{B}_{m}$ of $Z_{m}$ such that:

(a) $Z_{m}=Z_{m}^{\text {odd }}=Z_{m}^{\geq N}$

(b) For $z \in \mathcal{B}_{m}$,

$$
d_{m} z=\alpha+\sum_{z^{\prime}<z, z^{\prime} \in \mathcal{B}_{m}} \Phi_{z^{\prime}} z^{\prime}
$$

where $\Phi_{z^{\prime}} \in \Lambda^{+}(Y \oplus X)$ and $\alpha \in \Lambda^{\geq\lfloor N / 2\rfloor+1}(Y \oplus X)$. Here, $\lfloor K\rfloor$ denotes the greatest integer less than or equal to $K$.

For $m=0$, consider the model $\left(\Lambda X, d_{0}\right)$. By hypothesis $d X^{\text {even }} \subset \Lambda^{+} Y \otimes$ $\Lambda X$ and therefore $d_{0} X^{\text {even }}=0$. If $\left\{x_{1}, \ldots, x_{p}\right\}$ is a homogeneous basis for $X^{\text {even }}$, introduce new generators $\mathcal{B}_{0}=\left\{z_{1}, \ldots, z_{p}\right\}$, which will be the desired basis for $Z_{0}$, and define $d_{0} z_{j}=x_{j}^{\lfloor N / 2\rfloor+1}$. Well order $\mathcal{B}_{0}$ in any way. Then $\left(\Lambda X^{\text {even }} \otimes \Lambda Z_{0}, d_{0}\right)$ is elliptic and so $\left(\Lambda X \otimes \Lambda Z_{0}, d_{0}\right)$ is too (see [Ha1, $\S 6$ Prop. 1]). It is clear that (a) and (b) are satisfied.

By induction, assume we have built an elliptic KS-extension (and minimal model)

$$
\left(\Lambda\left(y_{m-1}, \ldots, y_{1}\right) \otimes \Lambda X \otimes \Lambda Z_{m-1}, d_{m-1}\right)
$$

and a well ordered basis $\mathcal{B}_{m-1}$ of $Z_{m-1}$ such that (a) and (b) are satisfied. We shall build $Z_{m}$ and $\mathcal{B}_{m}$ so that $\mathcal{B}_{m-1} \subset \mathcal{B}_{m}$ as ordered sets. To do so we will need to define $d_{m}$ (which we will call $d$ from now on) on $Z_{m-1}$. By previous remarks, it is enough to define $\theta_{m}$ (we denote it simply by $\theta$ ) on $Z_{m-1}$.

In order to define $\theta$ on $Z_{m-1}$, we'll need some new elements (on which we'll have to define $\theta$ as well), and we'll use a secondary induction on the well-ordering of $\mathcal{B}_{m-1}$ to do this.

To get this secondary induction started, note that it is clear by the (primary) assumption (b) for the first element $z_{0} \in \mathcal{B}_{m-1}$ that $\theta^{i}\left(d_{m-1} z_{0}\right)$ is defined for all $i$. Choose the least integer $r_{0}$ such that $\theta^{r_{0}+1} d_{m-1} z_{0}=0$. 
Now introduce new elements (whose dependence on $z_{0}$ we suppress) $U_{z_{0}}=$ $\left\{u_{1}, \ldots, u_{r_{0}}\right\}$ and define $\theta^{i}\left(z_{0}\right)=u_{i}, \theta\left(u_{r_{0}}\right)=0$, and $d_{m-1} u_{i}=\theta^{i} d_{m-1} z_{0}$ for $i=1, \ldots, r_{0}$. Note that this implies $\theta\left(u_{i}\right)=u_{i+1}$ for $i=1, \ldots, r_{0}-1$.

Now suppose by (the secondary) induction that, by adding the appropriate $U_{z^{\prime}}$ for $z^{\prime}<z$ as in the first step, we've already defined $\theta$ on $\left(\mathcal{B}_{m-1}\right)_{<z}$, as well as on these new elements. Recall that condition (b) for $z \in \mathcal{B}_{m-1}$ says that $d_{m-1} z \in \Lambda Y \otimes \Lambda X \otimes \Lambda Z_{<z}$, where $Z_{<z}$ denotes $\operatorname{span}\left\{\left(\mathcal{B}_{m-1}\right)_{<z}\right\}$. Since $\theta$ is already defined on $Z_{<z}$, we see that $\theta^{i} d_{m-1} z$ now makes sense for any $i$. We then proceed, as in the first step, to introduce new elements $U_{z}$ and define $\theta$ and $d_{m-1}$ on them. In this way, we also define $\theta^{i}(z)$ for any $i$, which closes the secondary induction.

Now set $\mathcal{B}_{m}=\mathcal{B}_{m-1} \cup \bigcup_{z \in \mathcal{B}_{m-1}} U_{z}$ and define a well ordering in $\mathcal{B}_{m}$ respecting the one in $\mathcal{B}_{m-1}$ and such that:

$$
z^{\prime}<u_{r_{z}}<\ldots<u_{1}<z
$$

where $z^{\prime}$ denotes the immediate predecessor of $z$.

It remains to prove that $\mathcal{B}_{m}$ and $Z_{m}$ satisfy (a) and (b). If $z \in \mathcal{B}_{m-1}$, then

$$
\begin{aligned}
d z & =d_{m-1} z+y_{m} \theta z \\
& =\beta+\sum_{z^{\prime}<z, z^{\prime} \in \mathcal{B}_{m-1}} \Psi_{z^{\prime}} z^{\prime}+y_{m} u_{1},
\end{aligned}
$$

with $\beta \in \Lambda^{\geq\lfloor N / 2\rfloor+1}(Y \oplus X)$ and $\Psi_{z^{\prime}} \in \Lambda^{+}(Y \oplus X)$. Thus, since $u_{1}<z$, (b) holds for each element of $\mathcal{B}_{m-1}$. Now, given $u_{i} \in U_{z}$, we have

$$
\begin{aligned}
d u_{i} & =d_{m-1} u_{i}+y_{m} \theta u_{i} \\
& =\theta^{i} d_{m-1} z+y_{m} u_{i+1} \\
& =\theta^{i}(\beta)+\theta^{i}\left(\sum_{z^{\prime}<z, z^{\prime} \in \mathcal{B}_{m-1}} \Psi_{z^{\prime}} z^{\prime}\right)+y_{m} u_{i+1} .
\end{aligned}
$$

But $u_{i+1}<u_{i}$ and $\theta^{p}\left(z^{\prime}\right)<z^{\prime}<u_{i}$, for $z^{\prime}<z$ and $p \geq 0$, while, by Remark 3.2, $\theta^{i}(\beta)=\gamma \in \Lambda^{\geq\lfloor N / 2\rfloor+1}(Y \oplus X)$. Therefore $d u_{i}$ can be written as

$$
d u_{i}=\gamma+\sum_{w<u_{i}, w \in \mathcal{B}_{m}} \Psi_{w} w
$$

with $\gamma \in \Lambda^{\geq\lfloor N / 2\rfloor+1}(Y \oplus X)$ and $\Psi_{w} \in \Lambda^{+}(Y \oplus X)$. This establishes (b) for the new elements of $\mathcal{B}_{m}$.

To prove (a) note that, since $d_{m-1} u_{i} \neq 0$ then, either $\gamma \neq 0$, or some $\Psi_{w} w \neq 0$. Remark 3.2 shows that the degree of $u_{i}$ is above $N$ in the first case 
while this is clear by an induction on the well-ordering of $\mathcal{B}_{m}$ in the second. Note that since the degree of $\theta$ is even $\mathcal{B}_{m}$, and hence $Z_{m}$, is concentrated in odd degrees.

To finish, observe that since $\left(\Lambda\left(y_{m-1}, \ldots, y_{1}\right) \otimes \Lambda X \otimes \Lambda Z_{m-1}, d_{m-1}\right)$ is elliptic, the minimal extension (and model) $\left(\Lambda\left(y_{m}, \ldots, y_{1}\right) \otimes \Lambda X \otimes \Lambda Z_{m}, d_{m}\right)$ is also elliptic. This closes the induction on $\operatorname{dim} Y$ and establishes Proposition 3.1.

There is a simple inductive description of the basis $\mathcal{B}_{m}$ that is evident from the above. Indeed, $\mathcal{B}_{m}$ is the disjoint union $\bigcup_{z \in \mathcal{B}_{m-1}}\left\{z, \theta z, \theta^{2} z, \ldots, \theta^{n(z)} z\right\}$, so that $Z_{m}$ is a direct sum of $\operatorname{dim} Z_{m-1}$ irreducible $\theta$-modules. In view of this, it is easy to see that the degrees of the new generators satisfy $N \leq|z| \leq D N$, where $\max \left\{|x|: x \in X^{\text {even }}\right\}=2 D$. Thus an upper bound for the number of new generators, in terms of $\operatorname{dim} Y=n, \min \{|y|: y \in Y\}=r+1$, $\operatorname{dim} X^{\text {even }}=p$ and $s=\lfloor(N D-N) / r\rfloor$ is

$$
\operatorname{dim} Z \leq p s^{n} .
$$

This proves the corollary of the introduction.

\section{Examples and open questions.}

Consider the (non-elliptic) minimal model $\left(\Lambda\left(y_{1}, y_{2}, y_{3}, y_{4}, y_{5}, x\right), d\right)$ with $\left|y_{i}\right|=1, d y_{i}=0$ for $1 \leq i \leq 5$ and $d x=y_{1} y_{2} y_{3}+y_{1} y_{4} y_{5}$. For $N=2$, the algorithm implicit in the proof of Proposition 3.1 gives us the following elliptic extension: $\left(\Lambda(Y, x) \otimes \Lambda\left(z_{0}, z_{1}, z_{2}, z_{3}\right), d\right)$ where

$$
\begin{aligned}
d z_{0} & =x^{2}+y_{3} z_{1}+y_{5} z_{3} \\
d z_{1} & =2 x y_{2} y_{3}+y_{5} z_{2} \\
d z_{2} & =2 y_{1} y_{2} y_{3} y_{4} \\
d z_{3} & =2 x y_{1} y_{4} .
\end{aligned}
$$

A simple example of a case not covered by Theorem 1 is the 5 -Postnikov stage of $\mathbf{S}^{2} \vee \mathbf{S}^{2}$ : Its model is $\left(\Lambda\left(x_{1}, x_{2}, y_{1}, y_{2}, y_{3}, x_{3}, x_{4}\right), d\right)$ with $\left|x_{1}\right|=\left|x_{2}\right|=$ $2, d y_{1}=x_{1}^{2}, d y_{2}=x_{2}^{2}, d y_{3}=x_{1} x_{2}, d x_{3}=x_{1} y_{3}-x_{2} y_{1}$, and $d x_{4}=x_{2} y_{3}-x_{1} y_{2}$. While $\left(\mathbf{S}^{2} \vee \mathbf{S}^{2}\right)^{5}$ does not belong to $\mathcal{M}$, Steve Halperin made the following observations. The model is quasi-isomorphic to $\left(\Lambda(a, b, y, c, e) /\left(a^{2}, b^{2}\right), d\right)$ with $|a|=|b|=2, d y=a b, d c=a y$, and $d e=b y$, Now define, for any $N$,

$$
\begin{aligned}
& d z_{0}=c^{N} a \\
& d z_{1}=c^{N+1} b-(N+1) z_{0} y b \text { and } \\
& d z_{2}=c^{2 N+2}-2(N+1) c^{N+1} z_{0} y+2(N+1) z_{0} z_{1} a .
\end{aligned}
$$


Since this renders $[c]$ nilpotent in $\left(\Lambda\left(c, z_{0}, z_{1}, z_{2}\right), \bar{d}\right)$, the model

$$
\left(\Lambda\left(a, b, y, c, z_{0}, z_{1}, z_{2}\right) /\left(a^{2}, b^{2}\right), \bar{d}\right)
$$

is elliptic [H2, Prop. 1]. One then mimics this construction to make $[e]$ nilpotent as well, adding another 3 odd generators. How one might do this systematically for higher stages of $\mathbf{S}^{2} \vee \mathbf{S}^{2}$ is still unclear to the authors.

\section{References}

[BL] H.J. Baues and J.-M. Lemaire, Minimal models in homotopy theory, Math. Ann., 255 (1977), 219-242.

[BG] A.K. Bousfield and V.K.A.M. Gugenheim, On PL De Rham theory and rational homotopy type, Memoirs of the A.M.S., 179(8) (1976).

[Br] W. Browder, Surgery on simply connected manifolds, Springer, 1972.

[F] Y. Félix, La Dichotomie Elliptique-Hyperbolique en Homotopie Rationnelle, Astérisque, 176 (1989).

[FH] John B. Friedlander and Stephen Halperin, An Arithmetic Characterization of the Rational Homotopy Groups of Certain Spaces, Inventiones Math., 53 (1979), 117133.

[G] P.P. Grivel, La suite spectral d'une fibration de Kan, C.R.Acad. Sci., Paris, 282 (1976), 1227-1229.

[H1] S. Halperin, Lectures on Minimal Models, Publ. Mémoires de la S.M.F., 9-10 (1983).

[H2] , Finiteness in the Minimal Models of Sullivan, Trans. Amer. Math. Soc., 230 (1977), 173-199.

[HL] S. Halperin and G. Levin, High skeleta of CW-complexes, L.N.M., 1183 (1986), 211-217.

[M] C. McGibbon, in discussion at the Fields Institute, May-June 1996.

[MW] C. McGibbon and C. Wilkerson, Loop spaces of finite complexes at large primes, Proc. Amer. Math. Soc., 96 (1986), 698-702.

[S] P. Selick, Moore conjectures, L.N.M., 1318 (1986), 219-227.

[Sp] M. Spivak, Spaces satisfying Poincaré duality, Topology, 6 (1967), 77-102.

[Su] Dennis Sullivan, Infinitesimal Computations in Topology, Publ. de l'I.H.E.S., 47 (1978), 269-331.

[T] Daniel Tanré, Homotopie Rationelle: Modèles de Chen, Quillen, Sullivan, Lecture Notes in Math., 1025, Springer-Verlag, 1983.

Received June 10, 1995 and revised November 17, 1996.

UNIVERSITY OF OTTAWA

OTtaWA, CANADA K1N6N5

E-mail address: bjessup@sciences.uottawa.ca

AND

Universidad de MÁlaga, Ap. 59

29080- Málaga, SPAIN

E-mail address: aniceto@agt.cie.uma.es 